\title{
Théorème de Division et Stabilité de Systèmes Holonomes
}

\author{
Par \\ Nguyen Tien DaI*
}

\section{$\S 0$. Introduction}

Depuis $\mathrm{R}$. Thom, traditionnellement le théorème de division est lié à la théorie de la stabilité des applications différentiables. La question de stabilité est résolue par la méthode de linéarisation. Elle consiste à réduire la question de la stabilité ordinaire à un problème linéaire de la stabilité infinitésimale. Dans cet article on veut généraliser cette méthode pour étudier la théorie de la stabilité de systèmes holonomes.

Le système holonome, par définition est un système microdifférentiel dont la variété caractéristique est une variété holonome (voir [1], [9]). La notion de la stabilité de systèmes holonomes apparaît pour la première fois dans [7], [8] où on donne la définition de déformations d'un réseau holonome vérifiant la condition de platitude. On obtient alors des bons résultats pour la classification de systèmes de Gauss-Manin généralisés dans [4].

Quand il s'agit de l'étude de la stabilité de systèmes holonomes en position générique (voir [6], Ch. I, §6), il est mieux d'utiliser les matrices génératrices (cf. [2]). Remarquons que la stabilité de matrices génératrices est un problème purement analytique qui généralise la stabilité d'applications analytiques au cas de matrices analytiques vérifiant certaines conditions. Donc on peut appliquer la méthode de linéarisation, en particulier la méthode d'approximations successives et le passage du "formel" au convergent pour démontrer "inf.-stable implique stable" qui est le point principal de ce travail.

C'est avec grand plaisir que je remercie Professeur Frédéric Pham qui m'a introduit la théorie de singularités de systèmes holonomes et Professeur Bernard Malgrange qui m'a donné de précieux conseils dans l'étude et la

Communique par M. Kashiwara, avril le 9, 1992.

1991 Mathematics Subject Classification: 35Q15

* Institute of Mathematics, P.O. Box 631, Boho 10.000, Hanoi, Vietnam. 
réalisation de ce problème.

\section{Théorème de Division et Applications}

\section{$\S 1$. Définitions et Notations}

1.1. Pour tout $n$-uple de variables $x=\left(x_{1}, \cdots, x_{n}\right), \boldsymbol{C}\{x\}=\boldsymbol{C}\left\{x_{1}, \cdots, x_{n}\right\}$ désignera l'algèbre des séries convergentes en $x_{1}, \cdots, x_{n}$.

Pour tout $A \in \mathbb{N}^{n}, A=\left(A_{1}, \cdots, A_{n}\right), x^{A}=x_{1}^{A_{1}} \cdots x_{n}^{A_{n}}$.

Pour tout $(A, i j) \in \mathbb{N}^{n} \times\{1, \cdots, m\}^{2}$,

$x^{A, i j}=\left(\begin{array}{cc}0 & 0 \\ x^{A} \\ \underbrace{0}_{m} & 0\end{array}\right)$, le monome étant à la $(i, j)$-ième place.

1.2. Définition. On appellera forme positive sur $\mathbb{R}^{n} \times\{1, \cdots, m\}^{2}$ un couple $L=(\widetilde{L}, \lambda)$ formé par une application linéaire $\widetilde{L}: \mathbb{R}^{n} \rightarrow \mathbb{R}$ à coefficients positifs non tous nuls $\left(l_{1}, \cdots, l_{n}\right)$, et une matrice $\lambda=\left(\lambda_{i j}\right) \in R_{+}^{m^{2}}$. On représentera la forme linéaire $L$ par le point $P$ de $\boldsymbol{R}_{+}^{n}$ de coordonnées $\left(l_{1}, \cdots, l_{n}\right)$. Une telle forme $L$ définit une application

$$
\begin{aligned}
& L: \boldsymbol{R}_{+}^{n} \times\{1, \cdots, m\}^{2} \rightarrow \boldsymbol{R}_{+} \\
& L\left(a_{1}, \cdots, a_{n} ; i j\right)=l_{1} a_{1}+\cdots+l_{n} a_{n}+\lambda_{i j}
\end{aligned}
$$

1.3. Remarque. On peut définir un bon ordre semi-lexicographique sur $\mathbb{N}^{n} \times\{1, \cdots, m\}^{2}$ noté $<$ de la manière suivante:

$$
\left(a_{1}, \cdots, a_{n} ; i j\right)<\left(b_{1}, \cdots, b_{n} ; r s\right)
$$

si et seulement si

$$
L\left(a_{1}, \cdots, a_{n}, i j\right)<L\left(b_{1}, \cdots, b_{n} ; r s\right)
$$

ou si

$$
L\left(a_{1}, \cdots, a_{n} ; i j\right)=L\left(b_{1}, \cdots, b_{n} ; r s\right)
$$

et soit $T(i j)<T(r s)$ où $T(i j) \equiv(i-1) \cdot m+j$

soit $(i j)=(r s)$ et il existe $p$ :

$0 \leqslant p \leqslant n$ tel que 
$a_{n}=b_{n}, \cdots, a_{p+1}=b_{p+1}$ et $a_{p}<b_{p}$.

Remarquons que $N^{n}$ agit sur $N^{n} \times\{1, \cdots, m\}^{2}$ de la manière suivante:

$$
A+(B ; i j)=(A+B ; i j) .
$$

1.4. Définition. Soit une matrice non nulle de séries convergentes sur C, $H=\left(h_{i j}\right) \in\{x\}^{m^{2}}$, on note

$$
h_{i j}=\sum_{A \in N^{n}} h_{A, i j} \cdot x^{A} ; i, j=1, \cdots, m .
$$

On appelle diagramme de Newton de $H$ l'ensemble d'indices

$$
Q(H)=\left\{(A, i j) \in N^{n} \times\{1, \cdots, m\}^{2}, h_{A, i j} \neq 0\right\} .
$$

On appelle exposant privilégié de $H$ pour la direction $L$ et on note $\exp _{L}(H)$ le plus petit élément de $Q(H)$ pour le bon ordre défini par $L$.

On dira que $H$ est monique si le coefficient du monôme de $H$ d'exposant $\exp _{L}(H)$ est égal à 1 .

1.5. Définition. Soit $M$ un sous-module de $C\{x\}^{m^{2}}$. On appelle ensemble des privilégiés de $M$ pour la direction $L$, le sous-ensemble de $N^{2} \times\{1, \cdots, m\}^{2}$ suivant:

$$
E_{L}(M)=\left\{\exp _{L}(H), H \in M\right\}
$$

Par les formules de multiplications on obtient

$$
E_{L}(M)+N^{n}=E_{L}(M) .
$$

Alors il existe une plus petite (pour l'inclusion) partie finie $F=F_{L}(M)$ de $E=E_{L}(M)$ telle que

$$
E=\bigcup_{a \in F} a+N^{n}
$$

On appelle $F$ l'escalier de $M$ pour la direction $L$.

1.6. Définition. On appellera partition associée au $p$-uple de $\boldsymbol{N}^{n} \times$ $\{1, \cdots, m\}^{2},\left[\left(A_{1}, i_{1} j_{1}\right), \cdots,\left(A_{p}, i_{p} j_{p}\right)\right]$, la partition suivante de $N^{n} \times\{1, \cdots, m\}^{2}$, l'une des parties pouvant éventuellement être vide: 
$1 \leqslant k \leqslant p:$

$$
\begin{aligned}
& \Delta_{k}=\left[\left(A_{k}, i_{k} j_{k}\right)+N^{n}\right] \backslash\left[\bigcup_{k^{\prime}<k}\left(A_{k^{\prime}}, i_{k^{\prime}} j_{k^{\prime}}\right)+N^{n}\right], \\
& \bar{\Delta}=\mathbb{N}^{n} \times\{1, \cdots, m\}^{2} \backslash \bigcup_{1 \leqslant k \leqslant p} \Delta_{k} .
\end{aligned}
$$

Notons aussi qu'une somme indixée par l'ensemble vide est nulle.

1.7. Définition. Pour tout poly-rayon $\rho \in\left(\mathbb{R}_{+}^{*}\right)^{n}, \boldsymbol{R}_{+}^{*}=\mathbb{R}_{+}-\{0\}$, on notera $\mathbb{C}(\rho)$ l'algèbre de séries convergentes en $n$ variables $x=\left(x_{1}, \cdots, x_{n}\right)$ :

$$
\boldsymbol{C}(\rho)=\left\{f=\sum_{A \in N^{n}} f_{A} \cdot x^{A}, f \in C(x\}, \sum\left|f_{A}\right| \cdot \rho^{A}<+\infty\right\}
$$

qui muni de la norme $\|f\|=\sum\left|f_{A}\right| \cdot \rho^{A}$, est une algèbre de Banach.

Sauf mention contraire, on munira l'espace de Banach $C(\rho)^{m}$ de la norme

$$
\left\|\left(f_{1}, \cdots, f_{m}\right)\right\|=\max _{1 \leqslant i \leqslant m}\left\|f_{i}\right\|
$$

et l'espace de Banach $C(\rho)^{m^{2}}$ de la norme

$$
\|H\|=\max _{1 \leqslant i \leqslant m} \sum_{j=1}^{m}\left\|h_{i j}\right\|
$$

Pour les démonstrations suivantes on utilise la notion d'un ouvert effilé dans [5]:

1.8. Définition (Dans [5], définition (1.1.11)). Soit $\tilde{L}$ une forme linéaire sur $\mathbb{R}^{n}$ de coefficients positifs non tous nuls $\left(l_{1}, \cdots, l_{n}\right)$. Nous dirons qu'un ouvert $V$ de $\mathbb{R}_{+}^{n}$ est effilé pour la direction $\tilde{L}$ s'il existe

$$
\begin{gathered}
\beta=\left(\beta_{1}, \cdots, \beta_{n}\right) \in\left(\boldsymbol{R}_{+}^{*}\right)^{n}, \\
\Lambda_{\beta}=\left\{\left(l_{1}+\delta_{1}, \cdots, l_{n}+\delta_{n}\right) \in\left(\mathbb{R}_{+}^{*}\right)^{n}, 0<\delta_{1}<\beta_{1} \delta_{2}<\cdots<\beta_{n-1} \delta_{n}<\beta_{n}\right\}
\end{gathered}
$$

et une fonction continue $C: \Lambda_{\beta} \rightarrow \mathbb{R}_{+}^{*}$ tels que l'ouvert $V$ contienne l'ouvert suivant de $\boldsymbol{R}_{+}^{n}$ : 


$$
\begin{gathered}
V_{\beta, C}=\left\{\left(\eta^{l_{j}^{\prime}}, \cdots, \eta^{l_{n}^{\prime}}\right) \in \mathbb{R}_{+}^{n},\left(l_{1}^{\prime}, \cdots, l_{n}^{\prime}\right) \in \Lambda_{\beta}, \eta \in \boldsymbol{R}_{+}\right\} \\
\text {et } \eta<C\left(l_{1}^{\prime}, \cdots, l_{n}^{\prime}\right) .
\end{gathered}
$$

\section{§2. Théorèmes de Divisions}

\subsection{Théorème de division par une famille de matrices.}

Soient $\tilde{L}$ une forme linéaire à coefficients positifs sur $\mathbb{R}^{n}$ et $\left(H^{1}, \cdots, H^{p}\right)$ des matrices non nulles de $\boldsymbol{C}\{x\}^{m^{2}}, x=\left(x_{1}, \cdots, x_{n}\right)$. Désignons par $\left(A_{k}, i_{k} j_{k}\right)$ l'exposant privilégié de $H^{k}(1 \leqslant k \leqslant p)$ pour la direction $L=(\tilde{L}, 0)$ et supposons $H^{k}$ monique. Notons $\left(\Delta_{k}, \bar{\Delta}\right)$ la partition associée au p-uple précédent. On a l'algorithme de division suivante:

1/ Pour tout $H \in \boldsymbol{C}\{x\}^{m^{2}}$ il existe des quotients uniques $g^{k} \in \boldsymbol{C}\{x\}, k=1, \cdots, p$ et un reste unique

$Q \in C\{x\}^{m^{2}}$ tels que

(i) $H=g^{1} \cdot H^{1}+\cdots+g^{p} \cdot H^{p}+Q$,

(ii) $g^{k}=\sum_{\left(A, i_{k} j_{k}\right) \in \Delta_{k}} g_{A}^{k} \cdot x^{A-A_{k}}(1 \leqslant k \leqslant p)$,

(iii) $Q=\sum_{(A, i j) \in \bar{\Delta}} q_{A, i j}, x^{A, i j}$

2/ Pour tout $a \in\left(\mathbb{R}_{+}^{*}\right)^{n}$, quand $m=1$ on peut prendre $a=0$, il existe un ouvert effilé pour la direction $\tilde{L}, V$ de $\left(\boldsymbol{R}_{+}^{*}\right)^{n}$ tel que pour tout poly-rayon $\rho \in V$ :

(i) si $H \in C(\rho)^{m^{2}}$ alors $g^{k} \in C(\rho), k=1, \cdots, p$ et $Q \in C(\rho)^{m^{2}}$ et

(ii) $\sum_{k=1}^{p}\left\|g^{k}\right\| \cdot \rho^{A_{k}}+\|Q\| \leqslant 2 m^{2} \cdot \rho^{-a} \cdot\|H\|$.

Démonstration. Au paragraphe (2.3).

\subsection{Théorème de division par un sous-module.}

Une forme linéaire positive $\tilde{L}$ sur $N^{n}$ et un sous-module $M$ de $C\{x\}^{m^{2}}$, $x=\left(x_{1}, \cdots, x_{n}\right)$ étant fixés, désignons par $E_{L}(\mu)$ et $F_{L}(\mu)$ l'ensemble des privilégiés de $\mu$ et l'escalier de $\mu$ pour la direction $L=(\tilde{L}, 0)$. Alors

1/ Toute famille $\left(H^{1}, \cdots, H^{p}\right)$ de matrices de $\mu$ telle que 


$$
F_{L}(\mu) \subset\left\{\exp _{L} H^{1}, \cdots, \exp _{L} H^{p}\right\}
$$

est un système de générateurs de $\mu$.

2/ Tout élément $H \in \mathbb{C}\{x\}^{m^{2}}$ est congru modulo $\mu$ à un unique élément de $\mathbb{C}\{x\}^{m^{2}}$ de la forme

$$
r(H)=\sum_{(A, i j) \notin E_{L}(\mu)} q_{A, i j} \cdot x^{A, i j}
$$

que nous appellerons reste de la division de $H$ par $\mu$. De plus pour tout $a \in\left(\mathbb{R}_{+}^{*}\right)^{n}$, (quand $m=1$ on peut prendre $a=0$ ) il existe un ouvert effilé $V=$ $V(a)$ pour la direction $\tilde{L}$ tel que pour tout poly-rayon $\rho \in V$ on ait:

$$
\begin{gathered}
r(H) \in \mathbb{C}\{\rho\}^{m^{2}} \text { si } H \in \mathbb{C}\{\rho\}^{m^{2}} \text { et } \\
\|r(H)\| \leqslant 2 m^{2} \cdot \rho^{-a} \cdot\|H\| .
\end{gathered}
$$

3/ Notons $\left(A_{k}, i_{k} j_{k}\right)(1 \leqslant k \leqslant p)$ les éléments de $F_{L}(\mu)$ et $Q_{k}$ le reste de la division de $x^{A_{k}, i_{k} j_{k}}$ par $\mu$. On appelle base standard de $\mu$, pour la direction $\widetilde{L}$, la famille $H_{k}=x^{A_{k}, i_{k} j_{k}}-Q_{k}, 1 \leqslant k \leqslant p$. Elle vérifie $\exp \left(H_{k}\right)=A_{k}, i_{k} j_{k}, H_{k} \in \mu$ et avec les notions de $2 /, \forall a \in\left(\mathbb{R}_{+}^{*}\right)^{n}, a=0$ si $m=1, \forall \rho \in V(a),\left\|H_{k}\right\| \leqslant 2 m^{2} \cdot \rho^{A_{k}-a}$.

4/ Il existe un ouvert effilé $V$ pour la direction $\widetilde{L}$ tel que pour tout $\rho \in V$ le sous-module de $\mathbb{C}\{\rho\}^{m^{2}} \mu(\rho)=\left\{H \in \mu\right.$ et $\left.H \in \mathbb{C}\{\rho\}^{m^{2}}\right\}$ est fermé et facteur directeur de $\mathbb{C}\{\rho\}^{m^{2}}$.

Preuve. Montrons comment ce théorème se déduit du précédent.

L'unicité de 2/ s'obtient de la manière suivante: s'il existait deux éléments distincts $Q$ et $Q^{\prime}$ de la forme du reste et congrus modulo $\mu$, par différence on aurait:

$$
\begin{aligned}
& Q-Q^{\prime}=\sum_{(A, i j) \notin E_{L}(\mu)}\left(q_{A, i j}-q_{A, i j}^{\prime}\right) x^{A, i j} \\
& Q-Q^{\prime} \neq 0 \text { d'où } \exp _{L}\left(Q-Q^{\prime}\right) \in E_{L}(\mu)
\end{aligned}
$$

et $\exp _{L}\left(Q-Q^{\prime}\right) \notin E_{L}(\mu)$ ce qui est absurde.

Pour toute famille $\left(H^{1}, \cdots, H^{p}\right)$ de matrices de $\mu$, l'algorithme de division permet d'écrire tout $H \in \mathbb{C}\{x\}^{m^{2}}$ :

$$
H=\sum_{k=1}^{p} g^{k} \cdot H^{k}+\sum_{(A, i j) \in \bar{\Delta}} q_{A, i j} \cdot x^{A, i j}
$$


si $\left\{\exp H^{1}, \cdots, \exp H^{p}\right\} \supset F_{L}(\mu)$,

$\bar{\Delta} \cap E_{L}(\mu)=\phi$, le reste de la division de $H$ par $H^{1}, \cdots, H^{p}$ est alors le reste de la division de $H$ par $\mu$; en particulier il est nul si $f \in \mu$. L'inégalité sur les normes est celle du théorème 2.1 .

Le 3/ est immédiat.

Le 4/ s'obtient en remarquant que $\mu(\rho)$ est le supplémentaire topologique du sous-espace:

$$
H(\rho)=\left\{Q=\sum_{(A, i j) \notin E_{L}(\mu)} q_{A, i j} \cdot x^{A, i j} \in C\{\rho\}^{m^{2}}\right\} \text { dans } \boldsymbol{C}\{\rho\}^{m^{2}}
$$

\subsection{Démonstration du théorème 2.1 .}

Avec les notations de (1.7) on munit le module libre $\boldsymbol{C}\{\rho\}^{m^{2}}$ de la famille de normes suivantes qui sont toutes uniformément équivalentes: pour tout $\alpha=\left(\alpha_{i j}\right)_{i, j=1, \cdots, m} \in\left(\boldsymbol{R}_{+}^{n}\right)^{m^{2}}$, on pose pour tout

$$
\begin{aligned}
& H=\left(h_{i j}\right) \in \boldsymbol{C}\{\rho\}^{m^{2}} \\
& N_{\alpha}(H)=\sum_{i=1}^{m} \sum_{j=1}^{m}\left\|h_{i j}\right\| \cdot \rho^{\alpha_{i j}}
\end{aligned}
$$

si $\rho_{j}<1,(1 \leqslant j \leqslant n)$ on a:

$$
\inf \left(\rho^{\alpha_{i j}}\right) \cdot \max \left\|h_{i j}\right\| \leqslant N_{\alpha}(H) \leqslant m^{2} \cdot \max \left\|h_{i j}\right\| .
$$

Muni de $N_{\alpha}, \boldsymbol{C}\{\rho\}^{m^{2}}$ est un espace de Banach que nous noterons $\boldsymbol{C}(\rho, \alpha)$.

2.3.1. Lemme. $\left(\bar{\Delta}, \Delta_{k}(1 \leqslant k \leqslant p)\right)$ ayant été définie en (1.6). Pour tous $\rho \in\left(\boldsymbol{R}_{+}^{*}\right)^{n}, \alpha \in\left(\boldsymbol{R}_{+}^{n}\right)^{m^{2}}$ considérons les espaces de Banach suivants:

$$
\begin{aligned}
& C(\Delta)=\left\{Q \in C(\rho, \alpha), Q=\sum_{(A, i j) \in \bar{\Delta}} q_{A, i j} x^{A, i j}\right\} \\
& C\left(\Delta_{k}\right)=\left\{g^{k} \in C(\rho), g^{k}=\sum_{\left(A, i_{k} j_{k}\right) \in \Delta_{k}} g_{A}^{k} x^{A-A_{k}}\right\}
\end{aligned}
$$

$H(\rho, \alpha)=\bigoplus_{k=1}^{p} C\left(\Delta_{k}\right) \oplus C(\bar{\Delta})$ muni de la norme suivante: 


$$
\left\|\left(g^{1}, \cdots, g^{p} ; Q\right)\right\|=\sum_{k=1}^{p}\left\|g^{k}\right\| p^{A_{k}+\alpha_{i_{k} j_{k}}}+\|Q\|
$$

Le morphisme

$$
\begin{aligned}
\varphi_{1}: H(\rho, \alpha) & \rightarrow C(\rho, \alpha) \\
\left(g^{1}, \cdots, g^{p} ; Q\right) & \mapsto \sum_{k=1}^{p} g^{k} x^{A_{k}, i_{k} j_{k}}+Q
\end{aligned}
$$

est une isométrie déspaces de Banach.

Preuve. Évidente.

Soient $\left(l_{1}, \cdots, l_{n}\right)$ les coefficients de la forme linéaire $\tilde{L}$ et $a \in\left(\boldsymbol{R}_{+}^{*}\right)^{n}$. Si $m=1$, on prend $a=0$ et on choisit $\lambda=\lambda_{11}=0$. Si $m \neq 1$, en utilisant la proposition (1.1.10) et le lemme (1.3.2) dans [5] on peut démontrer les résultats correspondants dans notre cas: c'est-à-dire il existe une constante strietement positive $\alpha$ et si on choisit $\lambda=\left(\lambda_{i j}\right) \in\left(\boldsymbol{R}_{+}\right)^{m^{2}}$ tels que

$$
\begin{gathered}
\lambda_{i j}<\lambda_{r s} \text { si } T(i j)<T(r s) \\
\text { et } \lambda_{m m}<\inf \left\{\alpha, l_{1} a_{1}+\cdots+l_{n} a_{n}\right\}
\end{gathered}
$$

on a

2.3.2. Lemme. Il existe des nombres réels strictement positifs $\beta_{1}, \cdots, \beta_{n}$, un ouvert non vide de $\left(\boldsymbol{R}_{+}^{*}\right)^{n}$

$$
\Lambda=\Lambda\left(\beta_{1}, \cdots, \beta_{n}\right)=\left\{\left(l_{1}+\delta_{1}, \cdots, l_{n}+\delta_{n}\right), 0<\delta_{1}<\beta_{1} \delta_{2}<\cdots<\beta_{n-1} \delta_{n}<\beta_{n}\right\}
$$

et une fonction continue $b: \Lambda \rightarrow \boldsymbol{R}_{+}$tels que:

Pour tout $P^{\prime} \in \Lambda$ représentant une forme linéaire $\tilde{L}^{\prime}$ et $L^{\prime}=\left(\tilde{L}^{\prime}, \lambda\right)$ on ait $L^{\prime}(B, i j)-L^{\prime}\left(A_{k}, i_{k} j_{k}\right) \geqslant b\left(P^{\prime}\right)$ pour chaque $k$ compris entre l et $p$ et chaque $(B, i j)$ supérieur à $\left(A_{k}, i_{k} j_{k}\right)$ pour l'ordre défini par la forme positive $L=(\tilde{L}, 0)$ sur $N^{n} \times\{1, \cdots, m\}^{2}$.

2.3.3. Perturbation de $\varphi_{1}$. Nous utiliserons les notations suivantes: $\tilde{L}^{\prime}$ désignera une forme linéaire réprésentée par un élément

$$
P^{\prime}=\left(l_{1}^{\prime}, \cdots, l_{n}^{\prime}\right) \in \Lambda ; L^{\prime}=\left(\tilde{L}^{\prime}, \lambda\right)
$$


À toute $\tilde{L}^{\prime}$ on associe une solution $\alpha^{\prime}=\left(\alpha_{i j}^{\prime}\right) \in\left(\boldsymbol{R}_{+}^{n}\right)^{m^{2}}$ du système d'équations $\tilde{L}^{\prime}\left(\alpha_{i j}^{\prime}\right)=\lambda_{i j}(i, j=1, \cdots, m)$ et à toute $\tilde{L}^{\prime}$ et à tout nombre réel positif $\eta$ on associe le poly-rayon $\rho^{\prime}=\left(\eta^{l_{1}^{\prime}}, \cdots, \eta^{l_{n}^{\prime}}\right)$. Remarquons, qu'avec ces notions, pour tout multi-indice

$$
(A, i j) \in \boldsymbol{N}^{n} \times\{1, \cdots, m\}^{2} \text { on a l'égalité }
$$

$$
\eta^{L^{\prime}(A, i j)}=\rho^{\prime A} \cdot \rho^{\prime \alpha_{i j}^{\prime}}
$$

On choisit un nombre réel positif $\eta_{0}$ tel que pour tout $P^{\prime} \in \Lambda$ les matrices $H^{1}, \cdots, H^{p}$ soient dans $\boldsymbol{C}\left(\rho_{0}^{\prime}\right)^{m^{2}}$, donc dans $\boldsymbol{C}\left(\rho_{0}^{\prime}, \alpha^{\prime}\right)$.

Comme $\left(A_{k}, i_{k} j_{k}\right)$ est l'exposant privilégié de $H^{k}, 1 \leqslant k \leqslant p$, et que $H^{k}$ a été supposé monique, on a la décomposition suivante:

$$
H^{k}=x^{A_{k}, i_{k} j_{k}}+U_{k}
$$

avec

$$
U_{k}=\sum_{(A, i j)>\left(A_{k}, i_{k} j_{k}\right)} h_{A, i j}^{k} \cdot x^{A, i j}
$$

Alors pour tout $\eta \in \boldsymbol{R}_{+}, \eta \leqslant \eta_{0}$, pour toute forme linéaire $\tilde{L}^{\prime}$ représentée par un point $P^{\prime} \in \Lambda, P^{\prime}$ et $\alpha^{\prime}$ associés à $\tilde{L}^{\prime}$ et à $\eta$, et pour tout $k$, on a $U_{k} \in \boldsymbol{C}\left(\rho^{\prime}, \alpha^{\prime}\right)$.

On peut donc considérer le morphisme

$$
\begin{aligned}
\varphi_{2}: H\left(\rho^{\prime}, \alpha^{\prime}\right) & \rightarrow C\left(\rho^{\prime}, \alpha^{\prime}\right) \\
\left(g^{1}, \cdots, g^{p} ; Q\right) & \mapsto \sum_{k=1}^{p} g^{k} \cdot U_{k}
\end{aligned}
$$

2.3.4. Lemme. Il existe une fonction $C: \Lambda \rightarrow R_{+}^{*}$ définissant l'ouvert effilé pour la direction $\tilde{L}$ :

$$
V=\left\{\rho^{\prime}=\left(\eta^{l_{1}^{\prime}}, \cdots, \eta^{l_{n}^{\prime}}\right) \in\left(\boldsymbol{R}_{+}^{*}\right)^{n},\left(l_{1}^{\prime}, \cdots, l_{n}^{\prime}\right) \in \Lambda ; 0<\eta \leqslant \eta_{0} \text { et } \eta<C\left(P^{\prime}\right)\right\}
$$

telle que pour tout $\rho^{\prime} \in \vee$ la norme du morphisme $\varphi_{2}$ correspondant soit inférieure à $\frac{1}{2}$

Preuve. Nous allons majorer la norme de $\varphi_{2}$ et définir la fonction 
continue $C$ au cours du calcul suivant:

$$
\begin{aligned}
\left\|\varphi_{2}\right\|= & \sup \frac{\left\|\sum g^{k} U_{k}\right\|}{\sum_{k=1}^{p}\left\|g^{k}\right\| \cdot \rho^{\prime A_{k}+\alpha_{i_{k} j_{k}}^{\prime}}+\|Q\|} \\
& \leqslant p \cdot \max _{1 \leqslant k \leqslant p}\left(\left\|U_{k}\right\| \cdot \rho^{\prime-A_{k}-\alpha_{i_{k} j_{k}}^{\prime}}\right)
\end{aligned}
$$

or

$$
\begin{gathered}
\left\|U_{k}\right\| \cdot \rho^{\prime-A_{k}-\alpha_{i_{k}}^{\prime} j_{k}}=\sum\left|f_{A, i j}^{k}\right| \cdot \eta^{L^{\prime}(A, i j)-L^{\prime}\left(A_{k}, i_{k} j_{k}\right)} \\
L^{\prime}(A, i j)>L^{\prime}\left(A_{k}, i_{k} j_{k}\right)
\end{gathered}
$$

d'après le lemme 2.3.2, $L^{\prime}(A, i j)-L^{\prime}\left(A_{k}, i_{k} j_{k}\right) \geqslant b\left(P^{\prime}\right)$ d'où

$$
\begin{aligned}
\left\|U_{k}\right\| \cdot \rho^{\prime-A_{k}-\alpha_{i_{k} y_{k}}^{\prime}} \leqslant & \left(\frac{\eta}{\eta_{0}}\right)^{b\left(P^{\prime}\right)} \cdot\left\|U_{k}\right\|_{C\left(\rho_{0}^{\prime}, \alpha^{\prime}\right)} \rho_{o}^{\prime-A_{k}-\alpha_{i_{k} j_{k}}^{\prime}} \\
& \leqslant \frac{1}{2 p} \operatorname{si} \eta \leqslant C\left(P^{\prime}\right) \in R_{+}^{*}
\end{aligned}
$$

où l'on définit la fonction continue sur $\Lambda, P^{\prime} \mapsto C\left(P^{\prime}\right)$, par l'égalité suivante:

$$
C\left(P^{\prime}\right)^{b\left(P^{\prime}\right)}=\frac{1}{2 p} \eta_{0}^{b\left(P^{\prime}\right)} \cdot \min _{\substack{1 \leqslant k \leqslant p \\ U_{k} \neq 0}}\left[\eta_{0}^{L^{\prime}\left(A_{k}, i_{k} j_{k}\right)}\left(\left\|U_{k}\right\|_{C\left(\rho_{0}^{\prime}, \alpha^{\prime}\right)}\right)\right]^{-1}
$$

On conclut que: si $\rho^{\prime} \in V$ alors $\left\|\varphi_{2}\right\| \leqslant \frac{1}{2}$.

\subsubsection{Comme l'isométrie}

$$
\varphi_{1}: H\left(\rho^{\prime}, \alpha^{\prime}\right) \rightarrow C\left(\rho^{\prime}, \alpha^{\prime}\right)
$$

entre ces mêmes espaces de Banach est de conorme égale à un, nous venons de montrer que le morphisme de division pour la famille $\left(H^{1}, \cdots, H^{p}\right)$ :

$$
\begin{aligned}
\varphi_{1}+\varphi_{2}: H\left(\rho^{\prime}, \alpha^{\prime}\right) & \rightarrow C\left(\rho^{\prime}, \alpha^{\prime}\right) \\
\left(g^{1}, \cdots, g^{p} ; Q\right) & \mapsto H=g^{1} H^{1}+\cdots+g^{p} H^{p}+Q
\end{aligned}
$$


est un isomorphisme de norme inférieure ou égale à 2 .

Au début de la démontration du théorème nous avions choisis $\lambda_{i j}$ tel que

$$
\lambda_{i j}<l_{1} a_{1}+\cdots+l_{n} a_{n},(i, j=1, \cdots, m)
$$

on en déduit que pour tout

$$
\rho^{\prime}=\left(l_{1}^{\prime}, \cdots, l_{n}^{\prime}\right) \in \Lambda, \lambda_{i j}<l_{1}^{\prime} a_{1}+\cdots+l_{n}^{\prime} a_{n},
$$

donc pour tout $\eta \in \boldsymbol{R}_{+}^{*}$ et $\eta<1$ :

$$
\rho^{\prime \alpha_{i j}^{\prime}}=\eta^{\lambda_{i j}}>\eta^{l_{1}^{\prime} a_{1}+\cdots+l_{n}^{\prime} a_{n}}=\rho^{\prime a} .
$$

Par ailleurs nous avions remarqué que la norme habituelle de $C\left\{\rho^{\prime}\right\}^{m^{2}}$ et la norme $N_{\alpha}$, sont reliées par les inégalités suivantes, pour tout $E=\left(e_{i j}\right) \in \boldsymbol{C}\left\{\rho^{\prime}\right\}^{m^{2}}$ :

$$
\max _{1 \leqslant i, j \leqslant m}\left\|e_{i j}\right\| \cdot \rho^{\prime a} \leqslant N_{\alpha \prime}(E) \leqslant m^{2} \cdot \max _{1 \leqslant i, j \leqslant m}\left\|e_{i j}\right\|
$$

On en déduit l'inégalité cherchée, avec les normes habituelles

$$
\sum_{k=1}^{p}\left\|g^{k}\right\| \cdot \rho^{A_{k}}+\|Q\| \leqslant 2 \cdot m^{2} \cdot p^{\prime-a} \cdot\|H\|
$$

Le théorème de division dans $C\left\{x_{1}, \cdots, x_{n}\right\}^{m^{2}}$ s'obtient par passage à la limite inductive sur $\rho^{\prime}$.

\subsection{Corollaire. Le morphisme $\varphi$ défini par}

$$
\varphi: \boldsymbol{C}\{x\}^{r} \rightarrow \boldsymbol{C}\{x\}^{m^{2}}, x=\left(x_{1}, \cdots, x_{n}\right)
$$

admet une scission $\psi$ C-linéaire

$$
\psi: C\{x\}^{m^{2}} \rightarrow C\{x\}^{r}
$$

vérifiant $\varphi \circ \psi \circ \varphi=\varphi$.

De plus il existe $a \in \boldsymbol{R}_{+}^{n}$ et un ouvert efflé $V$ de $\boldsymbol{R}_{+}^{n}$ tels que pour tout $\rho \in V, \varphi$ et $\psi$ induisent $\bar{\varphi}$ et $\bar{\psi}$ :

$$
\boldsymbol{C}(\rho)^{r} \underset{\bar{\psi}}{\stackrel{\bar{\varphi}}{\rightleftarrows}} \boldsymbol{C}\{\rho\}^{m^{2}}
$$


avec $\bar{\varphi} \circ \bar{\psi} \circ \bar{\varphi}=\bar{\varphi}$ et $\|\bar{\varphi}\| \leqslant \rho^{-a}$.

Preuve. Soient $E_{1}, \cdots, E_{r}$ les images par $\varphi$ des vecteurs de la base canonique de $\boldsymbol{C}\{x\}^{r}$, notons $\mu=\operatorname{Im} \varphi$ le sous-module de $\mathbb{C}\{x\}^{m^{2}}$ qu'ils engendrent. On choisit une forme positive $L=(\tilde{L}, 0)$ sur $\mathbb{R}^{n} \times\{1, \cdots, m\}^{2}$ et une famille $\left(H^{1}, \cdots, H^{p}\right)$ d'éléments de $\mu$ telle que les exposants privilégiés de $H^{k}$ : $\exp _{L}\left(H^{k}\right), 1 \leqslant k \leqslant p$, décrivent l'escalier $F_{L}(\mu)$ de $\mu$; on fixe ensuite des éléments $\lambda_{j}^{k}$ de $\mathbb{C}\{x\}$ tels que

$$
H^{k}=\sum_{j=1}^{r} \lambda_{j}^{k} \cdot E_{j}, k=1, \cdots, p .
$$

D'après le théorème $(2.2)$ toute $H \in C\{x\}^{m^{2}}$ s'écrit de manière unique et $C$-linéaire

$$
H=\sum_{j=1}^{r}\left(\sum_{k=1}^{p} g^{k} \cdot \lambda_{j}^{k}\right) \cdot E_{j}+Q
$$

$g^{k}$ et $Q$ vérifiant certaines conditions et $Q=0$ si, et seulement si, $H \in \mu=\operatorname{Im} \varphi$.

Posons

$$
\psi(H)=\left(\sum_{k=1}^{p} g^{k} \cdot \lambda_{j}^{k}, 1 \leqslant j \leqslant r\right)
$$

$\psi$ ainsi définie est une scission de $\varphi$. Pour obtenir la majoration, on applique le théorème (2.2) et on "grossit" $a$ pour se dispensen d'écrire les coefficients $2 m^{2}$ et $\left\|\lambda_{j}^{k}\right\|$.

\section{§3. Applications}

Nous utiliserons le théorème de division pour résoudre certains systèmes matriciels d'équations analytiques.

3.1. Enoncés de théorème. Soient

$$
z=\left(z_{1}, \cdots, z_{q}\right), y=\left(y_{1}, \cdots, y_{q^{\prime}}\right), \phi=\left(\phi_{1}, \cdots, \phi_{r}\right),
$$

trois familles de variables et $R \in \mathbb{C}\{z, y, \phi\}^{m^{2}}$. 
On appelle solution du système d'équations analytiques $(R), \varphi \in C\{z, y\}^{r}$ avec $\varphi(0,0)=0$ et tel qu'en substituant dans $R$ on ait

$$
R(z, y, \varphi)=0 .
$$

On appelle solution à l'ordre $n \in N$ le $\varphi_{n} \in \boldsymbol{C}\{z, y\}^{r}$, avec $\varphi(0,0)=0$ et tel qu'en substituant dans $R$ on ait:

$$
R\left(z, y, \varphi_{n}\right) \in m^{n+1} \cdot C\{z, y\}^{m^{2}},
$$

$m$ désignant l'idéal maximal de $\boldsymbol{C}\{z\}$. Remarquons que si $\varphi_{n}^{\prime}$ est congru modulo $\left(m^{n+1}\right)$ à une solution à l'ordre $n \varphi_{n}$ alors $\varphi_{n}^{\prime}$ est aussi solution à l'ordre $n$.

On dit qu'une solution à l'ordre $n+1 \varphi_{n+1}$ prolonge une solution à l'ordre $n \varphi_{n}$ si les deux solutions sont congrus modulo $\left(m^{n+1}\right)$.

Théorème. Tout système d'équations analytiques $(R)$ admettant une solution à l'ordre $n_{0}$ et tel que pour tout entier $n \geqslant n_{0}$, toute solution à l'ordre $n$ qui prolonge la solution à l'ordre $n_{0}$ donnée se prolonge en une solution à l'ordre $n+1$, admet une solution convergente qui prolonge la solution à l'ordre $n_{0}$ donnée.

\subsection{Construction de la solution.}

Quitte à effectuer un changement de variables qui transformerait

$$
R(z, y, \phi) \text { en } R\left(z, y, \phi-\varphi_{o}\right)
$$

on peut supposer que $\varphi(0, y) \equiv 0$ est solution à l'ordre 0 .

Une solution à l'ordre $n, \varphi_{n}$ se prolonge en une solution à l'ordre $n+1$ si et seulement si il existe $\gamma_{n+1} \in\left(\boldsymbol{C}\{y\}[z]_{n+1}\right)^{r}$, où $\boldsymbol{C}\{y\}[z]_{n+1}$ désignant l'ensemble de polynomes homogènes de degré $n+1$ en $z$, tel que $\varphi_{n}+\gamma_{n+1}$ soit solution à l'ordre $n+1$.

Une solution $\varphi_{n}$ à l'ordre $n$ étant choisie, on notera $R_{n+1}$ le terme homogène de degré $n+1$ en $z$ dans $\left(C\{y\}[z]_{n+1}\right)^{m^{2}}$ de

$$
R\left(z, y, \varphi_{n}\right) \in\left(m^{n+1} \cdot C\{y\}\right)^{m^{2}}
$$

3.2.1. Désignons par $A_{i}$ les matrices suivantes à coefficients dans $C\{y\}$ :

$$
A_{i}=\frac{\partial R}{\partial \phi_{i}}(0, y, 0), i=1, \cdots, r
$$


Posons

$$
\widetilde{R}(z, y, \phi) \equiv R(z, y, \phi)-\sum_{i=1}^{r} A_{i} \phi_{i}
$$

les égalités suivantes sont satisfaites:

$$
\begin{aligned}
& \widetilde{R}(0, y, 0)=0 \\
& \frac{\partial \widetilde{R}}{\partial \phi_{i}}(0, y, 0)=0, i=1, \cdots, r .
\end{aligned}
$$

Pour $\phi=\left(\phi_{1}, \cdots, \phi_{r}\right), A=\left(A_{1}, \cdots . A_{r}\right)$ on pose

$$
A \cdot \phi=\sum_{i=1}^{r} A_{i}, \phi_{i}
$$

3.2.2. Lemme. Pour tout $n \in N$, considérons l'application

$$
\begin{aligned}
\beta_{n}:\left(\boldsymbol{C}\{y\}[z]_{n}\right)^{r} \rightarrow\left(\boldsymbol{C}\{y\}[z]_{n}\right)^{m^{2}} \\
\gamma_{n} \mapsto A \cdot \gamma_{n} .
\end{aligned}
$$

Une solution $\varphi_{n}$ à l'ordre $n$ se prolonge en une solution à l'ordre $n+1$ si et seulement si $R_{n+1} \in \operatorname{Im}\left(\beta_{n+1}\right)$.

Dans ce cas si

$$
R_{n+1}=-\beta_{n+1}\left(\gamma_{n+1}\right)
$$

alors $\varphi_{n}+\gamma_{n+1}$ est solution à l'ordre $n+1$.

Preuve. À l'aide de la formule de Taylor et en négligeant les termes d'ordres supériers à $n+1$, on exprime que

$$
R\left(z, y, \varphi_{n}\right) \in m^{n+1}\left(C\{y\}^{m^{2}}\right)
$$

et que

$$
R\left(z, y, \varphi_{n}+\gamma_{n+1}\right) \in m^{n+2}\left(C\{y\}^{m^{2}}\right) .
$$

Alors on a le lemme. 
Soient

$$
\begin{aligned}
\beta: C\{y\}^{r} & \rightarrow C\{y\}^{m^{2}} \\
\gamma & \mapsto A \cdot \gamma .
\end{aligned}
$$

et $\varepsilon$ la scission de $\beta$ construite en (2.4).

On définit:

$$
\beta_{n}\left(\sum_{|A|=n} \delta_{A}(y) z^{A}\right)=\sum_{|A|=n} \beta\left(\delta_{A}(y)\right) z^{A}
$$

donc $\varepsilon_{n}:\left(C\{y\}[z]_{n}\right)^{m^{2}} \rightarrow\left(C\{y\}[z]_{n}\right)^{r}$

$$
\varepsilon_{n}\left(\sum_{|A|=n} \theta_{A}(y) z^{A}\right)=\sum_{|A|=n} \varepsilon\left(\theta_{A}(y)\right) \cdot z^{A}
$$

est une scission de $\beta_{n}$.

3.2.3 Construction de la solution. On définit par récurrence sur l'entier $n$, la suite $\left(\varphi_{n}\right)$ de solutions à l'ordre $n$ qui se prolongent l'une l'autre, par les relations:

$$
\begin{aligned}
\varphi_{0} & =0, \\
\gamma_{n+1} & =-\varepsilon_{n+1}\left(R_{n+1}\left(z, y, \varphi_{n}\right)\right), \\
\varphi_{n+1} & =\varphi_{n}+\gamma_{n+1}, \\
\varphi_{n+1} & =\gamma+\cdots+\gamma_{n+1} .
\end{aligned}
$$

Proposition. Soient $c \in \boldsymbol{R}_{+}^{*}, c<1$ et $(c, \cdots, c) \in\left(\boldsymbol{R}_{+}^{*}\right)^{q+q^{\prime}+r}$ le poly-rayon d'un poly-disque sur lequel converge un représentant de $R$ dans $C\{z, y, \phi\}^{m^{2}}$. Il existe deux poly-rayons $\rho \in\left(\boldsymbol{R}_{+}^{*}\right)^{q}$ et $\mu \in\left(\boldsymbol{R}_{+}^{*}\right)^{q \prime}$ tel que

(i) $|\rho|<c$ et $|\mu|<c$;

(ii) $\forall n \in N, \varphi_{n} \in C(\rho, \mu)^{r}$

$$
\left\|\varphi_{n}\right\|_{C(\rho, \mu)}<c
$$

La proposition implique que la serie $\sum_{n=1}^{\infty} \gamma_{n}$ converge dans $\boldsymbol{C}(\rho, \mu)^{r}$; soit $\varphi$ sa somme.

Alons $\varphi \in \boldsymbol{C}\{z, y\}^{r}$ est solution du système $(R)$ et prolonge la solution nulle 
$\varphi_{0}=0$ fixée initialement. La démonstration du théorème est donc ramenée à la démontration de la proposition.

\subsection{Démonstration de la proposition.}

3.3.1. Choix $d u$ poly-rayon $\mu$ : A l'aide du corollaire (2.4) on fixe $\mu \in\left(\boldsymbol{R}_{+}^{*}\right)^{q \prime}$ tel que $|\mu|<c$. $\quad \beta$ et $\varepsilon$ induisent des morphismes d'espaces de Banach entre $\mathbb{C}(\mu)^{r}$ et $\mathbb{C}(\mu)^{m^{2}}$.

Soit $N$ la norme de $\varepsilon$.

Pour tout $n \in N$ et pour tout $\rho \in\left(\boldsymbol{R}_{+}^{*}\right)^{q}, \beta_{n}$ et $\varepsilon_{n}$ induisent des morphismes

$$
C(\rho, \mu)^{r} \underset{\varepsilon^{n}}{\stackrel{\beta n}{\rightleftarrows}} C(\rho, \mu)^{m^{2}}
$$

d'espaces de Banach. La norme de $\varepsilon_{n}$ est $N$.

Comme $\gamma_{n+1}=-\varepsilon_{n+1}\left(R_{n+1}\right), \quad R_{n+1}=-A \cdot \gamma_{n+1}, \quad$ on obtient $\left\|\gamma_{n}\right\| \leqslant N$. $\left\|A \cdot \gamma_{n}\right\|$ puis en sommant

$$
\begin{aligned}
\left\|\varphi_{n+1}\right\| & =\left\|\gamma_{1}\right\|+\cdots+\left\|\gamma_{n+1}\right\| \\
& \leqslant N \cdot\left\|R_{n+1}\right\|+N \cdot\left\|A \cdot \varphi_{n}\right\| \\
& =N \cdot\left\|R_{n+1}-\left(A \cdot \varphi_{n}\right)\right\| .
\end{aligned}
$$

3.3.2. Série majorante auxiliaire. Notons $F$ la série en $\rho=\left(\rho_{1}, \cdots, \rho_{q}\right)$ et $v$ à coefficients réels positifs définie de la facon suivante:

Soit $\left(\widetilde{R}_{i j}\right)_{i, j=1, \cdots, m}$ un représentant de $\widetilde{R}$ dans $C\{z, y, \phi\}^{m^{2}}$ qui converge sur le poly-disque de poly-rayon $(c, \cdots, c)$ :

$$
\begin{aligned}
& \widetilde{R}_{i j}=\sum_{H, J, K} \widetilde{R}_{i j, H J K} z^{H} y^{J} \phi^{K} \\
& F(\rho, v)=\sum_{i, j=1}^{m} \sum_{H, J, K}\left|\widetilde{R}_{i j, H J K}\right| \mu^{J} \rho^{H} v^{|K|} .
\end{aligned}
$$

Cette série converge dans le poly-disque de poly-rayon $(c, \cdots, c)$ et satisfait aux relations suivantes:

$$
\begin{aligned}
& \|\widetilde{R}\|_{C(\rho, \mu,(v, \cdots, v))^{m^{2}}} \leqslant F(\rho, v), \\
& F(0,0)=0,
\end{aligned}
$$




$$
\frac{\partial F}{\partial v}(0,0)=0
$$

3.3.3. Les deux dernières relations provenant de (3.2.1). Grâce à ces relations il existe $\eta_{1} \in \boldsymbol{R}_{+}^{*}, \eta_{1}<\frac{c}{q}$ et il existe deux séries en $\eta$ et $v, H_{1}(\eta, v)$ et $H_{2}(\eta, v)$ convergentes sur le poly-disque de poly-rayon $\left(\eta_{1}, c\right)$ telles que $N \cdot F(\rho(\eta), v)=\eta^{6} \cdot H_{1}(\eta, v)+v^{2} \cdot H_{2}(\eta, v)$, où on pose $\rho(\eta)=\left(\eta^{6}, \cdots, \eta^{6}\right) \in\left(\boldsymbol{R}_{+}^{*}\right)^{q}$, $0<\eta<\eta_{1}$.

Posons

$$
\begin{aligned}
H(\eta, \omega) & \equiv \eta^{-3} \cdot N \cdot F\left(\rho(\eta), \eta^{3} \omega\right) \\
& =\eta^{3} \cdot H_{1}\left(\eta, \eta^{3} \omega\right)+\eta^{3} \cdot \omega^{2} \cdot H_{2}\left(\eta, \eta^{3} \omega\right)
\end{aligned}
$$

où $v=\eta^{3} \cdot \omega$.

Alors la fonction $\omega-H(\eta, \omega)$ vérifie les conditions du théorème des fonctions implicites. Donc il existe $\eta_{0}, 0<\eta_{0}<\eta_{1}$, tel que la suite définie par récurrence $\omega_{0}=0, \omega_{n+1}=H\left(\eta_{0}, \omega_{n}\right)$ soit bien définie et que $\omega_{n}<c$ pour tout $\eta$.

Donc on fixe $\rho=\rho\left(\eta_{0}\right) \in\left(\boldsymbol{R}_{+}^{*}\right)^{q}$ et la suite $v_{n}=\eta_{0}^{3} \cdot \omega_{n}$ vérifient les conditions:

$$
|\rho|<c, v_{n}<c \text { et } v_{n+1}=N \cdot F\left(\rho, v_{n}\right)
$$

3.3.4. On peut démontrer par récurrence sur $n$ que

$$
\left\|\varphi_{n}\right\|_{C(\rho, \mu)} \leqslant v_{n}<c
$$

En effet, $\varphi_{0}=0$, supposons l'assertion vraie pour $n$, on obtient

$$
\widetilde{R}\left(z, y, \varphi_{n} \in C(\rho, \mu)\right.
$$

puis, d'après (3.3.1) et (3.3.3)

$$
\begin{aligned}
\left\|\varphi_{n+1}\right\|_{\boldsymbol{C}(\rho, \mu)} & \leqslant N \cdot\left\|\widetilde{R}\left(z, y, \varphi_{n}\right)\right\|_{\boldsymbol{C}(\rho, \mu)} \\
& \leqslant N \cdot\|\widetilde{R}\|_{\boldsymbol{C}\left(\rho, \mu,\left(v_{n}, \cdots, v_{n}\right)\right)} \\
& \leqslant N \cdot F\left(\rho, v_{n}\right)=v_{n+1}<c .
\end{aligned}
$$

Donc on a la proposition. 


\section{Stabilité de Systèmes Holonomes}

\section{$\S 1$. Matrices Génératrices du Système Holonome}

Soit $X=\mathbb{C}^{n+1} \ni \bar{x}=\left(x_{0}, x_{1}, \cdots, x_{n}\right)$ une variété analytique complexe, dont nous noterons $T^{*} X \rightarrow X$ le fibré cotangent et $P^{*} X \rightarrow X$ le fibré projectif associé. Soit $M$ un germe de système microdifférentiel holonome au point $\left(0, d x_{0}\right) \in P^{*} X$, c'est-à-dire un module cohérent sur l'anneau des opérateurs microdifférentiels (d'ordre fini) dont la variété caractéristique est un sous-ensemble analytique dans $P^{*} X$ de la codimension $n+1$. Par une transformation de contact convenable de $P^{*} X$ à lui-même, la variété caractéristique de $M$ admet un système de paramètres de la forme $x_{1}, \cdots, x_{n}$ (cf. [6], Ch. I. §6). Dans ce cas on dit que le système $M$ est en position générique. Dans toute la suite le mot système holonome signifiera "système holonome en position générique".

On note $\mathscr{E}_{X}\left[\right.$ resp. $\left.\mathscr{E}_{X}^{(0)}\right]$ l'anneau noethérien des germes d'opérateurs microdifférentiels d'ordre fini [resp. d'ordre 0], et $\mathscr{E}_{x}^{\prime}\left[\right.$ resp. $\left.\mathscr{E}_{X}^{\prime(0)}\right]$ le sous-anneau (noethérien, commutatif) des operateurs dont le symbole total ne dépend que de $\xi_{0}, x_{1}, \cdots, x_{n}$. Soit $R$ un "réseau" de $M$, c'est-à-dire un sous-Ë ${ }_{X}^{(0)}$-module noethérien engendrant $M$ sur $\mathscr{E}_{X}$.

Supposons que les conditions du théorème 5.9 dans [7] sont satisfaites, alors il existe une base $u_{1}, \cdots, u_{m}$ de $R$ sur $\mathscr{E}_{X}^{\prime(0)}$ telle que on a les présentation suivante:

$$
\left\{\begin{array}{l}
x_{0} u=\left(A_{0}(x)+A_{1} \xi_{0}^{-1}\right) u \\
\xi_{j} \xi_{0}^{-1} u=B_{j}(x) u, j=1, \cdots, n
\end{array}\right.
$$

où $A_{1}$ est une matrice constante de la forme de Jordan.

Les matrices $A_{0}(x), B_{i}(x), i=1, \cdots, n$ analytiques en $x=\left(x_{1}, \cdots, x_{n}\right)$ vérifient les conditions d'intégrabilité:

$$
\begin{aligned}
& {\left[A_{0}(x), B_{i}(x)\right]=\left[B_{i}(x), B_{j}(x)\right]=0} \\
& -\frac{\partial A_{0}(x)}{\partial x_{i}}=\left[A_{1}, B_{i}(x)\right]+B_{i}(x) \\
& \frac{\partial B_{i}(x)}{\partial x_{j}}=\frac{\partial B_{j}(x)}{\partial x_{i}}, i, j=1, \cdots, n
\end{aligned}
$$

Il est facile de montrer qu'il existe une unique matrice $H(x)$ telle que 


$$
\begin{aligned}
& \text { trac } H(x)=0, \\
& {\left[A_{1}, H(0)\right]+H(0)=-A_{0}(0)}
\end{aligned}
$$

et

$$
\frac{\partial H(x)}{\partial x_{i}}=B_{i}(x), i=1, \cdots, n
$$

On appelle $H(x)$ la matrice génératrice du système holonome $M$ (correspondante à la base $u_{1}, \cdots, u_{m}$ ).

Les conditions d'intégrabilité se traduisent alors par les relations suivantes:

$$
\begin{aligned}
& {\left[\left[A_{1}, H(x)\right]+H(x), \frac{\partial H}{\partial x_{i}}\right]=0,} \\
& {\left[\frac{\partial H}{\partial x_{i}}, \frac{\partial H}{\partial x_{j}}\right]=0, i, j=1, \cdots, n .}
\end{aligned}
$$

\section{§2. Stabilité de Systèmes Holonomes}

La notion de la stabilité d'un réseau du système holonome a d'abord été énoncée par Pham [8]. Dans ce paragraphe on ètudie la stabilité du système holonome par celle de sa matrice génératrice.

On rappelle les définitions données dans [2]. Soit $H(x), x=\left(x_{1}, \cdots, x_{n}\right)$ $\in C^{n}$ une matrice génératrice du système $M$. On dit que la matrice génératrice $\bar{H}(x, y)$ du système $\bar{M}$ est une déformation de $H(x)$ de la base $y \in C^{k}$ si elle vérifie $\bar{H}(x, 0)=H(x)$.

La déformation $\bar{H}(x, y)$ de $H(x)$ est dite triviale s'il existe $\varphi:\left(C^{n} \times C^{k}, 0\right) \rightarrow$ $\left(\boldsymbol{C}^{n}, 0\right)$ avec $\varphi(x, 0) \equiv x$ tel que

$$
\bar{H}(x, y)=H(\varphi(x, y)) .
$$

La matrice génératrice $H(x)$ est dite stable si toute déformation de $H$ est triviale.

Une déformation $H^{*}(x, y)$ de $H(x)$ de la base $y \in C^{k}$ est dite verselle si toute déformation $\bar{H}(x, z)$ de $H(x)$ de la base $z \in C^{p}$ se laisse mettre sous la forms

$$
\bar{H}(x, z)=H^{*}(\varphi(x, z), \psi(z)),
$$

où $\varphi:\left(\boldsymbol{C}^{n} \times \boldsymbol{C}^{p}, 0\right) \rightarrow\left(\boldsymbol{C}^{n}, 0\right)$ avec $\varphi(x, 0) \equiv 0$, et $\psi:\left(\boldsymbol{C}^{p}, 0\right) \rightarrow\left(C^{k}, 0\right)$ avec 
$\psi(0)=0$.

Il est traditionnel depuis J.Mather d'étudier la stabilité d'un germe d'application defférentiable par la méthode de linéarisation. Elle consiste à réduire la question de la stabilité ordinaire à un problème linéaire de la stabilité infinitésimale. Ici on applique cette méthode pour étudier la stabilité de la matrice génératrice du système holonome.

On donne une définition de la stabilité infinitésimale.

Définition. La matrice génératrice $H(x)$ est dite infinitésimalement stable si toute déformation $\bar{H}(x, z)$ de la base $z \in \mathscr{Z}$, où d'idéal maximal de $O_{\mathscr{Z}}$ est de carré nul et de dimension finie sur $C$, est triviale.

Théorème de stabilité. La matrice génératrice $H(x)$ est stable si et seulement si elle est infinitésimalement stable.

\section{Démonstration.}

Stable $\Rightarrow$ inf. stable. Évidente.

Inf. stable $\Rightarrow$ Stable.

Soit $H(x)$ infinitésimalement stable.

Soit $\bar{H}(x, z)$ une déformation de $H(x)$ de la base $z \in C^{k}$.

On veut construire le morphisme

$$
\begin{aligned}
\varphi:\left(C^{n} \times C^{k}, 0\right) & \rightarrow\left(C^{n}, 0\right) \\
(x, z) & \mapsto \varphi(x, z) \quad \text { avec } \varphi(x, 0) \equiv x
\end{aligned}
$$

tel que

$$
\bar{H}(x, z)=H(\varphi(x, z))
$$

On établit le système d'équations analytiques

$$
R(x, z, \varphi) \equiv \bar{H}(x, z)-H(\varphi(x, z)) .
$$

Pour résoudre la solution $\varphi(x, z)$ de ce système on utilise le théorème 3.1 dans les applications.

Pour simplifier on note

$$
X=C^{n}, Z=C^{k}, Y=X \times Z,
$$

$m=\left(z_{1}, \cdots, z_{k}\right)$ l'idéal maximal de $O_{\mathscr{X}}$. Pour tout $n \in N$ on définit 


$$
\begin{aligned}
& Z_{n}=V\left(m^{n}\right)=\operatorname{Spec}\left(O_{\mathscr{Z}} / m^{n}\right), \\
& Y_{n}=\underset{Z}{Y \times Z_{n}}=X \times Z_{n} .
\end{aligned}
$$

On a le diagramme suivant:

$$
\begin{aligned}
& X=Y_{0} \hookrightarrow \cdots \hookrightarrow Y_{n} \hookrightarrow Y_{n+1} \cdots \hookrightarrow Y \\
& \downarrow \quad \downarrow \downarrow \downarrow \downarrow \\
& \{0\}=z_{0} \hookrightarrow \cdots \varsigma Z_{n} \hookrightarrow Z_{n+1} \cdots \hookrightarrow Z
\end{aligned}
$$

où les flèches verticales sont les projections.

Alors la solution $\varphi$ du système $(R)$ est la rétraction de $c X \subseteq Y$ dans le diagramme suivant

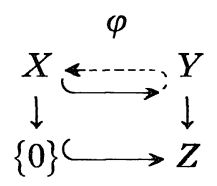

qui vérifie $R(x, z, \varphi,(x, z)) \equiv 0$.

La solution $\varphi_{n}$ à l'ordre $n \in N$ est la rétraction de $X \hookrightarrow Y_{n}$ dans le diagramme suivant

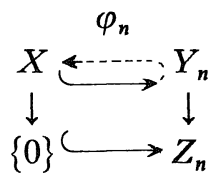

qui vérifie $R\left(x, z, \varphi_{n}\right) \in m^{n+1} \cdot O_{Y}^{m^{2}}$.

Pour $n=0$ on prend $\varphi_{0}(x) \equiv x$.

D'après le théorème 3.1 il suffit de montrer que pour tout $n \in N$ la solution $\varphi_{n}$ à l'ordre $n$ peut prolonger en une solution $\varphi_{n+1}$ à l'ordre $n+1$. D'après le lemme 3.2.3 dans [5], pour tout $n \in N$ on peut définir les produit fibrés.

$$
\begin{aligned}
& O_{X_{n+1}}=O_{X} \underset{O_{X_{n}}}{\times O_{Y_{n+1}}} \\
& O_{S_{n+1}}=C \underset{O_{Z n}}{\times} O_{Z_{n+1}}
\end{aligned}
$$

Il est clair que l'idéal maximal de $O_{S_{n+1}}$ est de carré nul et de dimension 
finie sur $\boldsymbol{C}$.

Comme $H(x)$ est infinitésimalement stable il existe $\phi_{n+1}$ dans le diagramme suivant

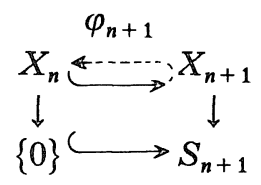

tel que $R\left(x, z, \phi_{n+1}\right)=0 \in O_{X_{n+1}}^{m^{2}}$.

En appliquant la propriété universelle des produits fibrés $O_{X_{n+1}}$ et $O_{S_{n+1}}$, le morphisme $\phi_{n+1}$ induit $\varphi_{n+1}$ dans le diagramme suivant

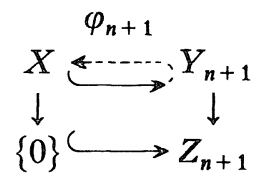

tel que

$$
R\left(x, z, \varphi_{n+1}\right)=0 \in O_{Y_{n+1}}^{m^{2}}
$$

c'est-à-dire

$$
R\left(x, z, \varphi_{n+1}\right) \in m^{n+2} \cdot O_{Y}^{m^{2}} .
$$

Le théorème de stabilité est ainsi démontré.

\section{§3. Critère de Stabilité}

Nous allons interpréter la stabilité infinitésimale d'une matrice génératrice $H(x), x=\left(x_{1}, \cdots, x_{r}\right)$ à l'aide de ses dérivées $\frac{\partial H(x)}{\partial x_{i}}, i=1, \cdots, r$ qui doivent vérifier les conditions suivantes

$$
\begin{aligned}
& {\left[\left[A_{1}, H(x)\right]+H(x), \frac{\partial H}{\partial x_{i}}\right]=0} \\
& {\left[\frac{\partial H}{\partial x_{i}}, \frac{\partial H}{\partial x_{j}}\right]=0, i, j=1, \cdots, r} \\
& \left(A_{1}\right. \text { est une matrice constante). }
\end{aligned}
$$


Notons $b$ le sous-module de $C\{x\}^{m^{2}}$ engendré par les matrices $\frac{\partial H(x)}{\partial x_{i}}$, $i=1, \cdots, r$ c'est-à-dire

$$
b=\left\{B(x)=\sum_{i=1}^{r} b_{i}(x) \frac{\partial H(x)}{\partial x_{i}}, b_{i}(x) \in C\{x\}\right\}
$$

Il est clair que $b$ est commutatif.

On dit que le sous-module $b$ est meximal si $b$ contienne toutes les matrices dans $\boldsymbol{C}\{x\}^{m^{2}}$ qui commutent avec $\frac{\partial H(x)}{\partial x_{i}}, i=1, \cdots, r$.

3.1. Remarque. Il est facile de montrer que si la sous-algèbre $b_{0}=\left\{B=\sum_{i=1}^{r} b_{i} \frac{\partial H(0)}{\partial x_{i}}, b_{i} \in C\right\}$ est commutative maximale dans $C^{m^{2}}$ alors $b$ est maximal.

On a un critère de stabilité suivant:

3.2. Proposition. Si b est maximal, $H(x)$ est stable.

Démonstration. Grâce au théorème de stabilité il suffit de démontrer que $H(x)$ est infinitésimalement stable, c'est-à-dire pour tout $n \in N$ on vent trouver $\phi_{n+1}$ tel que dans le diagramme suivant

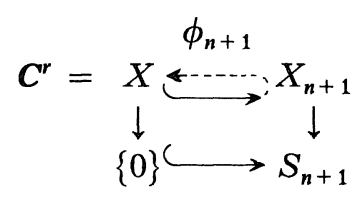

on a $R\left(x, z, \phi_{n+1}\right)=0 \in O_{X_{n+1}}^{m^{2}}$ (avec les notations dans la démonstration du théorème de stabilité).

Pour $n \in N$ on suppose qu'il existe une solution $\varphi_{n}$ à l'ordre $n$ telle que

$$
R\left(x, z, \varphi_{n}\right) \in m^{n+1} \cdot C\{x, z\}^{m^{2}}
$$

c'est-à-dire

$$
\bar{H}(x, z)-H_{n}(x, z)=0 \bmod m^{n+1} \cdot C\{x, z\}^{m^{2}}
$$

où on note $H_{n}(x, z) \equiv H\left(\varphi_{n}(x, z)\right)$. 
Alors leurs développements tayloriens en $z$ coincident jusqu'aux termes de degré $n$ inclus, c.a.d si

$$
\begin{aligned}
& \bar{H}(x, z)=\sum_{l=0}^{\infty} \bar{H}^{l}(x, z), \bar{H}^{l}(x, z)=\sum_{|\alpha|=l} \bar{H}_{\alpha}^{l}(x) z^{\alpha}, \\
& H_{n}(x, z)=\sum_{l=0}^{\infty} H_{n}^{l}(x, z), H_{n}^{l}(x, z)=\sum_{|\alpha|=l} H_{n \alpha}^{l}(x) z^{\alpha},
\end{aligned}
$$

alors

$$
\bar{H}^{l}(x, z)=H_{n}^{l}(x, z), l=0,1, \cdots, n,
$$

où $H(x)=\bar{H}^{0}(x, z)=H_{n}^{0}(x, z)$.

D'autre part, puisque $\bar{H}(x, z)$ et $H_{n}(x, z)$ sont les matrices génératrices alors pour tout $i=1, \cdots, r$ et $j=1, \cdots, k$ on a les relations

$$
\left[\frac{\partial \bar{H}}{\partial x_{i}}, \frac{\partial \bar{H}}{\partial z_{j}}\right]=\left[\frac{\partial H_{n}}{\partial x_{i}}, \frac{\partial H_{n}}{\partial z_{j}}\right]=0
$$

En comparant les coefficients de degré $n$ en $z$ de ces équations et compte tenu des relations précédentes on obtient

$$
\left[\frac{\partial H(x)}{\partial x_{i}}, \frac{\partial\left[\bar{H}^{n+1}(x, z)-H_{n}^{n+1}(x, z)\right]}{\partial z_{j}}\right]=0
$$

pour tout $i=1, \cdots, r$ et $j=1, \cdots, k$.

Donc

$$
\left[\frac{\partial H(x)}{\partial x_{i}}, \bar{H}^{n+1}(x, z)-H_{n}^{n+1}(x, z)\right]=0, i=1, \cdots, r
$$

Comme $b$ est maximal alors il existe les fonctions $h_{n+1}^{i}(x, z), i=1, \cdots, r$ homogènes de degré $n+1$ en $z$ telles que

$$
\bar{H}^{n+1}(x, z)-H_{n}^{n+1}(x, z)=\sum_{i=1}^{r} h_{n+1}^{i}(x, z) \frac{\partial H(x)}{\partial x_{i}} .
$$

Pour la solution d'ordre $n$

$$
\varphi_{n}=\left(\varphi_{n}^{1}(x, z), \cdots, \varphi_{n}^{r}(x, z)\right) \in \boldsymbol{C}\{x, z\}^{r}
$$


on définit la solution d'ordre $n+1$

$$
\varphi_{n+1}=\left(\varphi_{n+1}^{1}(x, z), \cdots, \varphi_{n+1}^{r}(x, z)\right) \in C\{x, z\}^{r}
$$

avec

$$
\varphi_{n+1}^{i}(x, z)=\varphi_{n}^{i}(x, z)+h_{n+1}^{i}(x, z), i=1, \cdots, r
$$

Si on note $H_{n+1}(x, z) \equiv H\left(\varphi_{n+1}(x, z)\right)$, alors par un calcul direct on obtient

$$
\bar{H}(x, z)-H_{n+1}(x, z)=0 \bmod m^{n+2} \cdot \boldsymbol{C}\{x, z\}^{m^{2}}
$$

ou

$$
R\left(x, z, \varphi_{n+1}\right) \in m^{n+2} \cdot C\{x, z\}^{m^{2}}
$$

Donc, $\varphi_{n}$ s'est ainsi prolongé en $\varphi_{n+1}$.

L'application $\phi_{n+1}$ est définie par

$$
\begin{aligned}
\phi_{n+1}: X_{n+1} & \rightarrow X=C^{r} \\
(x, z) & \mapsto\left(h_{n+1}^{1}(x, z), \cdots, h_{n+1}^{r}(x, z)\right) .
\end{aligned}
$$

La proposition est démontrée.

3.3. Example. Dans [3] on construit la déformation verselle $H(x)$ d'une matrice génératrice $H_{0}\left(x_{1}\right)$ du type $A_{r+1}$ :

$$
H_{0}\left(x_{1}\right)=x_{1} \cdot X+\frac{1}{2} x_{1}^{2} \cdot(Y)^{r}
$$

où
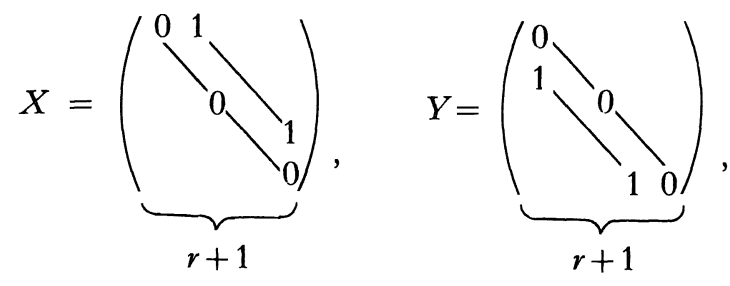


$$
(\mathrm{Y})^{k}=\underbrace{Y \cdots Y}_{k}, \quad k=1, \cdots, r
$$

et la matrice constante $A_{1}$ est dans la forme

$$
A_{1}=\operatorname{diag}\left(\frac{1}{r+2}, \cdots, \frac{r+1}{r+2}\right) .
$$

Tout d'abord on définit une matrice $A(x)$ par la formule

$$
A(x)=X+\sum_{k=1}^{r} \omega_{k}(x) \cdot(Y)^{k}
$$

où

$$
\begin{aligned}
& \omega_{k}(x)=\sum_{\alpha} Q_{k}(\alpha) x^{\alpha}, x^{\alpha}=x_{1}^{\alpha_{1}} \cdots x_{r}^{\alpha_{r}}, \\
& Q_{k}(\alpha)=\frac{1}{\alpha !} \prod_{j=1}^{s-1}(j(r+2)-k)
\end{aligned}
$$

avec

$$
\begin{aligned}
& \alpha_{1}+\cdots+\alpha_{r}=S, \\
& 2 \alpha_{r}+3 \alpha_{r-1}+\cdots+(\mathrm{r}+1) \alpha_{1}=\mathrm{k}+1 . \\
& \left(\text { si } S=1 \text { on pose } Q_{k}(\alpha)=1\right) .
\end{aligned}
$$

Il est facile de montrer les propriétés suivantes:

$$
\frac{\partial[A(x)]^{k}}{\partial x_{j}}=\frac{\partial[A(x)]^{j}}{\partial x_{k}}, \quad j, k=1, \cdots, r .
$$

Donc il existe unique matrice $H(x), x=\left(x_{1}, \cdots, x_{r}\right)$ déterminée par

$$
H(0)=0
$$

et

$$
\frac{\partial H(x)}{\partial x_{k}}=[A(x)]^{k}, k=1, \cdots, r
$$

La matrice génératrice $H(x)$ est stable par la remarque 3.1. En effet, 
l'algèbre $b_{0}$ engendrée par les matrices $(X)^{k}, k=1, \cdots, r$ est maximale.

\section{Bibliographie}

[ 1 ] Bjork, J.E., Rings of differential operators, North Holand Math., 1979.

[ 2 ] Dai, N.T., Les singularités de systèmes holonomes, à paraître.

[ 3 ] - The singularities of type $A_{k}$ of holonomic systems, en préparation.

[ 4 ] Dai, N.T., Duc, N.H. and Pham, F., Singularités non-gégénérées des systèmes de Gauss-Manin réticulés, Bull. Soc. Math. France, Mémoire 6, (1981).

[ 5 ] Galligo, A., Théorème de division et stabilité en géométrie analytique locale, Ann. Inst. Fourier, 29 (1979), 107-184.

[6] Kashiwara, M. and Kawai, T., On holonomic systems with regular singularities III, Publ. RIMS, Kyoto Univ., 17 (1981), 813-979.

[ 7 ] Malgrange, B., Déformations de systèmes différentiels et microdifférentiels, Séminaire E.N.S., 1979-1980.

[ 8 ] Pham, F., Déploiements de singularités de systèmes holonomes, C.R.A.S., 289 (1979), 333-336.

[ 9 ] Sato, M., Kawai, T. and Kashiwara, M., Microfunctions and pseudo-differential Equations, Lecture Notes in Math., 287 (1973). 
